\title{
Intrinsic Particle Charges and the Strong Force
}

\author{
Bo Lehnert \\ Alfvén Laboratory, Royal Institute of Technology, Stockholm, Sweden \\ Email: bo.lehnert@ee.kth.se
}

How to cite this paper: Lehnert, B. (2017) Intrinsic Particle Charges and the Strong Force. Journal of Modern Physics, 8, 10531066.

https://doi.org/10.4236/jmp.2017.87067

Received: May 12, 2017

Accepted: June 11, 2017

Published: June 14, 2017

Copyright (c) 2017 by author and Scientific Research Publishing Inc. This work is licensed under the Creative Commons Attribution International License (CC BY 4.0).

http://creativecommons.org/licenses/by/4.0/

\begin{abstract}
Starting from a revised quantum electrodynamic theory by the author, an attempt is made to elaborate a particle model of quarks which in their turn form triangular neutron and proton configurations. These "quark particles" are found to be electrically neutral but contain intrinsic electrical charges of both polarities, being an order of magnitude larger than the elementary charge, e. The main interaction force between two such particles is further found to have an attractive short-range character, and it becomes nearly two orders of magnitude larger than the repulsive force which would arise from two interacting elementary charges. The spatial potential distribution of this force corresponds to an inner barrier, an intermediate well, and an outer barrier. The well is found to have a depth being nearly equal to the binding energy 8 $\mathrm{MeV}$ of the neutron. The distribution of the barriers and the well makes a stable position possible for the mutual particle distance. The deduced radii of the outer shell and of the core are further of the same magnitude as the known nuclear radii of the neutron and proton. All these deduced characteristic features are the same as those of the known strong force concept. This raises the question whether the present results could provide a first step in a unification of the electromagnetic and the strong nuclear forces.
\end{abstract}

\section{Keywords}

Quantum Electrodynamics, Zero Point Energy, Intrinsic Particle Charges, Strong Force

\section{Introduction}

From the analysis of the harmonic oscillator, Planck proved the existence of a lowest nonzero energy level of the vacuum state, the Zero Point Energy (ZPE). This state generally includes photon-like electromagnetic oscillations, both with and without associated electric charges. It manifests itself in the Casimir force arising in vacuo between two metal plates separated by a small spacing [1]. This force is due to the imposed boundary conditions which lead to the disappear- 
ance of a small low-frequency part of the much larger ZPE photon spectrum. It has been shown by experiments to result in a real macroscopic pressure [2]. Consequently, the vacuum is not merely a state of empty space.

The ZPE and the Casimir force imply that there are sources in the vacuum which have to be included into the electromagnetic field equations. This is taken into account in an earlier Lorentz and gauge invariant treatise by the author [3] [4], including an incorporated four-current which is associated with a nonzero electric field divergence, $\operatorname{div} \boldsymbol{E}$. It results in broken symmetry of the electric and magnetic field strengths, $\boldsymbol{E}$ and $\boldsymbol{B}$. Here the nonzero $\operatorname{div} \boldsymbol{E}$ increases the degrees of freedom, acting somewhat like a hidden variable.

Spatial integration of $\operatorname{div} \boldsymbol{E}$ over the volumes of corresponding elementary particle models results both into nonzero and zero net electric charges, as well as into intrinsic charges of both polarities. The net charges occur in the case of divergent generating functions of the electromagnetic field strengths, thereby leading to point-charge-like solutions, but the intrinsic charges also occur in cases of convergent generating functions [3]. In this connection it should be observed that the quantized field equations become identical with the original equations in which the potentials and current densities are replaced by their expectation values. The resulting revised quantum electrodynamic theory (RQED) [3] [4] thus becomes an alternative to the conventional description of fundamental physics in terms of the Standard Model. Here it should be emphasized that the conventional form of Maxwell's equations is fully adequate in describing the dynamics of media containing already existing charged particles, such as a plasma. But the same equations can be questioned when considering the fundamental physics of the interior of the elementary particles. One example where Maxwell's equations fail in the vacuum state of an empty space is given by the photon which has to possess a nonzero spin [3].

The main purpose of this investigation is to show that the RQED theory can lead to interactions being the same as those due to the conventional strong force. The broken symmetry of RQED results in substantial intrinsic electric charges, even being an order of magnitude larger than the elementary charge, as will be seen in the following parts of this investigation. The mutual interaction force between the intrinsic charges of two particles is therefore expected to become both of short range and two orders of magnitude larger than that which arises from interaction between elementary charges. Such an intrinsic charge interaction thus has basic features being the same as those of the strong nuclear force described by Walker [5] among others. It can debouch into a speculation whether this interaction would interfere with the strong force, or even become an explanation of the latter [4] [6].

This paper presents a first attempt in studying the intrinsic charge effects and their relation to the strong force, in terms of a simple particle model based on a convergent generating function. It starts in Sections 2 and 3 with the restrictions and basic concepts of the present analysis, continues with the main features of a corresponding particle model in Section 4, and ends with a tentative quark mod- 
el of the proton and neutron in Section 5.

\section{Restrictions of Present Model}

With the main purpose of demonstrating the effects of the intrinsic charges, a first approach is thus made in terms of a convergent generating function of the electromagnetic field strengths. This avoids the additional complication of a divergent such function, with its related net point-charge behaviour and a related renormalisation process [3] [4].

There further exists a possibility of studying a compound particle model. This would include a divergent generating function with both net and intrinsic charges, superimposed on a convergent generating function with intrinsic charges only. Also this possibility is postponed to later studies.

The coming analysis will further be restricted to a relatively simple form of generating function, treated as an Ansatz representing a set of various geometrical profiles.

\section{Basic Equations}

A steady-state particle model will now be elaborated, to form a starting point of the present analysis. For detailed deductions of the underlying theory, reference is made to an earlier treatise [3]. A steady axisymmetric state is considered in a spherical frame $(r, \theta, \varphi)$, being independent of the angle $\varphi$ and having a current density $\boldsymbol{j}=(0,0, C \bar{\rho})$, an electric charge density $\bar{\rho}$, an electrostatic potential $\phi$, a magnetic vector potential $\boldsymbol{A}=(0,0, A)$, and a velocity vector $\boldsymbol{C}=(0,0, C)$, where $C= \pm c$ represents the two spin directions. The field equations then take the form

$$
\left(r_{0} \rho\right)^{2} \bar{\rho} / \varepsilon_{0}=D \phi=\left[D+(\sin \theta)^{-2}\right](C A)
$$

with $\rho=r / r_{0}$ where $r_{0}$ stands for a characteristic radial dimension and the operator $D$ has the form

$$
\begin{aligned}
& D=D_{\rho}+D_{\theta} \quad D_{\rho}=-\frac{\partial}{\partial \rho}\left(\rho^{2} \frac{\partial}{\partial \rho}\right) \\
& D_{\theta}=-\frac{\partial^{2}}{\partial \theta^{2}}-\frac{\cos \theta}{\sin \theta} \frac{\partial}{\partial \theta}
\end{aligned}
$$

With the generating function

$$
F(r, \theta)=C A-\phi=G_{0} G(\rho, \theta)
$$

having the amplitude $G_{0}$ and the dimensionless part $G$, self-consistent forms

$$
\begin{gathered}
\phi=-\left[1+(\sin \theta)^{2} D\right] F \\
C A=-(\sin \theta)^{2} D F \\
\bar{\rho}=-\left(\varepsilon_{0} / r_{0}^{2} \rho^{2}\right) D\left[1+(\sin \theta)^{2} D\right] F
\end{gathered}
$$

are obtained for the potentials and the local charge density. This makes the elec- 
tric and magnetic field strengths and the electric charge density interconnected through the generating function. For a volume element $\mathrm{d} V=2 \pi r^{2}(\sin \theta) \mathrm{d} \theta \mathrm{d} r$ the total integrated charge becomes

$$
q=2 \pi \varepsilon_{0} G_{0} r_{0} \int_{0}^{\infty \pi} f \mathrm{~d} \theta \mathrm{d} \rho
$$

where

$$
f(\rho, \theta)=-(\sin \theta) D\left[1+(\sin \theta)^{2} D\right] G
$$

The total self-force acting on the particle due to the fields $\boldsymbol{E}=-\nabla \phi$, $\boldsymbol{B}=\operatorname{curl} \boldsymbol{A}$ and the charge density $\bar{\rho}$ becomes [3]

$$
\boldsymbol{F}=\int_{0}^{\infty \pi} \int_{0} \bar{\rho}(\boldsymbol{E}+\boldsymbol{C} \times \boldsymbol{B}) \mathrm{d} V
$$

According to Section 0 we restrict the analysis to a convergent and separable generating function given by the Ansatz

$$
G(\rho, \theta)=R(\rho) \cdot T(\theta) \quad R=\rho^{\gamma} \mathrm{e}^{-\rho} \quad T=(\sin \theta)^{\alpha}
$$

with the disposable parameters $\gamma>0$ and $\alpha>0$, and where $G$ is finite for all $\rho$ and $\theta$. Various values of $\gamma$ and $\alpha$ represent various but similar profile shapes.

The present theory with its nonzero $\operatorname{div} \boldsymbol{E}$ leads to a nonzero particle rest mass, as given by [3] [7]

$$
m=\left(1 / c^{2}\right) \int \frac{1}{2} \bar{\rho}(\phi+C A) \mathrm{d} V
$$

With the function

$$
g=-\left[1+2(\sin \theta)^{2} D\right] G
$$

the same mass becomes

$$
m=\pi\left(\varepsilon_{0} / c^{2}\right) r_{0} G_{0}^{2} J_{m}
$$

where

$$
J_{m}=\int_{0}^{\infty \pi} f g \mathrm{~d} \rho \mathrm{d} \theta
$$

Treating the present particle model as a boson in a first attempt, the spin is given by [3] [7]

$$
\pi \varepsilon_{0}\left(C / c^{2}\right)\left(r_{0} G_{0}\right)^{2} J_{s}=h / 2 \pi
$$

where

$$
J_{s}=\int_{0}^{\infty \pi} \int_{0}^{\pi} \rho(\sin \theta) f g \mathrm{~d} \rho \mathrm{d} \theta
$$

Other forms of the spin would only lead to minor changes in the following numerical computations. Combination of relations (13) and (15) results in

$$
r_{0} m=(h / 2 \pi c)\left(J_{m} / J_{s}\right)
$$




\section{Main Features of Present Particle Model}

\subsection{The Electric Charge Density}

The charge density given by relations (7) and (8) can be combined with the identity

$$
\begin{aligned}
& \frac{\mathrm{d}}{\mathrm{d} \theta}\left\{(\sin \theta) \frac{\mathrm{d}}{\mathrm{d} \theta}\left[(\sin \theta)^{2}\left(D_{\theta} T\right)\right]+(\sin \theta) \frac{\mathrm{d} T}{\mathrm{~d} \theta}\right\} \\
& =-(\sin \theta)\left\{D_{\theta} T+D_{\theta}\left[(\sin \theta)^{2}\left(D_{\theta} T\right)\right]\right\}
\end{aligned}
$$

This results in a total integrated charge $q$ given by

$$
\begin{aligned}
q / 2 \pi \varepsilon_{0} G_{0} r_{0}= & \int_{0}^{\infty} \int_{0}^{\pi} R \frac{\mathrm{d}}{\mathrm{d} \theta}\left\{(\sin \theta) \frac{\mathrm{d}}{\mathrm{d} \theta}\left[(\sin \theta)^{2}\left(D_{\theta} T\right)\right]+(\sin \theta) \frac{\mathrm{d} T}{\mathrm{~d} \theta}\right\} \mathrm{d} \rho \mathrm{d} \theta \\
& -\int_{0}^{\infty} \int_{0}^{\infty}(\sin \theta)\left\{T+D_{\theta}\left[(\sin \theta)^{2} T\right]\right\} \frac{\mathrm{d}}{\mathrm{d} \rho}\left(\rho^{2} \frac{\mathrm{d} R}{\mathrm{~d} \rho}\right) \mathrm{d} \rho \mathrm{d} \theta \\
& -\int_{0}^{\infty \pi} \int_{0}(\sin \theta)^{3}\left\{T \frac{\mathrm{d}}{\mathrm{d} \rho}\left[\rho^{2} \frac{\mathrm{d}}{\mathrm{d} \rho}\left(D_{\rho} R\right)\right]+\left(D_{\theta} T\right) \frac{\mathrm{d}}{\mathrm{d} \rho}\left(\rho^{2} \frac{\mathrm{d} R}{\mathrm{~d} \rho}\right)\right\} \mathrm{d} \rho \mathrm{d} \theta
\end{aligned}
$$

For a convergent generating function with finite parts $R$ and $T$ as well as their derivatives, the total intrinsic charge $q$ then vanishes. This is because the partial integration in respect to $\theta$ in the first integral of Equation (19) disappears according to the identity (18), and because $\sin \theta=0$ for $\theta=0$ and $\pi$. The partial integration in respect to $\rho$ of the second and third integrals further leads to vanishing results since $R$ and its derivatives are zero at $\rho=0$ and $\infty$.

The local and radial intrinsic charge distribution

$$
q(\rho)=4 \pi \varepsilon_{0} G_{0} r_{0} \int_{0}^{\pi / 2} p(\rho, \theta) \mathrm{d} \theta
$$

with

$$
\begin{aligned}
p(\rho, \theta)= & -(\sin \theta)\left\{T+D_{\theta}\left[(\sin \theta)^{2} T\right]\right\} \rho^{2} \frac{\mathrm{d} R}{\mathrm{~d} \rho} \\
& -(\sin \theta)^{3}\left[T \rho^{2} \frac{\mathrm{d}}{\mathrm{d} \rho}\left(D_{\theta} R\right)+\left(D_{\theta} T\right) \rho^{2} \frac{\mathrm{d} R}{\mathrm{~d} \rho}\right]
\end{aligned}
$$

differs on the other hand from zero. It gives rise to a distribution of considerable intrinsic charges of both signs. With the form (10) and the integrals

$$
I_{m}=\int_{0}^{\pi / 2}(\sin \theta)^{m} \mathrm{~d} \theta
$$

the normalized radial distribution of charge density is then given by

$$
p(\rho)=\rho^{\gamma} \mathrm{e}^{-\rho}\left(k_{1} h_{1}+k_{2} h_{2}\right)
$$

where

$$
\begin{gathered}
k_{1}=\gamma(\gamma+1)-2(\gamma+1) \rho+\rho^{2} \\
k_{2}=-\gamma^{2}(\gamma+1)^{2}+4\left(1+3 \gamma+3 \gamma^{2}+\gamma^{3}\right) \rho \\
-2\left(7+9 \gamma+3 \gamma^{2}\right) \rho^{2}+4(\gamma+2) \rho^{3}-\rho^{4}
\end{gathered}
$$




$$
\begin{gathered}
h_{1}=\left(3+4 \alpha+2 \alpha^{2}\right) I_{\alpha+1}-2\left(3+3 \alpha+\alpha^{2}\right) I_{\alpha+3} \\
h_{2}=-I_{\alpha+3}
\end{gathered}
$$

\subsection{The Electromagnetic Field Strength}

The total electromagnetic field strength in Equation (9) consists both of an electric part $\boldsymbol{E}$ and of a magnetostatic part $\boldsymbol{C} \times \boldsymbol{B}$. With the potentials (4) and (5) it has the form

$$
\boldsymbol{E}^{*}=\boldsymbol{E}+\boldsymbol{C} \times \boldsymbol{B}=\left(G_{0} / r_{0}\right)\left(e_{r}^{*}, e_{\theta}^{*}, 0\right)
$$

with the corresponding normalized components

$$
e_{r}^{*}=e_{r}-C b_{\theta}=\frac{\partial G}{\partial \rho}-\frac{1}{\rho}(\sin \theta)^{2}(D G)
$$

and

$$
e_{\theta}^{*}=e_{\theta}+C b_{r}=\frac{\partial G}{\partial \theta}+(\cos \theta)^{2} \frac{\partial}{\partial \theta}(\sin \theta)^{2} D G-(\sin \theta)(\cos \theta)(D G)
$$

A substantial part of the contribution from $\boldsymbol{E}$ is here outbalanced by the contribution from $\boldsymbol{C} \times \boldsymbol{B}$ in the present geometry. The total field strength $\boldsymbol{E}^{*}$ vanishes only completely in some elementary geometrical configurations, such as in a straight cylindrical case and in that of interacting parallel line currents [3].

With the generating function (10) the radial part $e_{r}^{*}$ becomes symmetric in respect to the equatorial plane $\theta=\pi / 2$, whereas the polar part $e_{\theta}^{*}$ becomes antisymmetric in respect to the same plane. The radial part will be of special interest in the coming deductions. It reduces to the radial distribution

$$
e_{r}^{*}(\rho)=\rho^{\gamma-1} \mathrm{e}^{-\rho}\left[g_{1} I_{\alpha}+g_{2} I_{\alpha+2}\right]
$$

where

$$
\begin{gathered}
g_{1}=\gamma+\alpha^{2}-\rho \\
g_{2}=\gamma(\gamma+1)-\alpha(\alpha+1)-2(\gamma+1) \rho+\rho^{2}
\end{gathered}
$$

\subsection{The Electromagnetic Self-Force}

When considering the total integrated force $F$ of Equation (9), it has to be observed that the relations (18) and (19) cannot be applied to simplify the deductions. This is due to the appearance of the additional factor $\boldsymbol{E}^{*}$. Thus the full expression for $f(\rho, \theta)$ has to be used on the form given by

$$
\begin{aligned}
-f(\rho, \theta) /(\sin \theta)= & R\left(D_{\theta} T\right)+R D_{\theta}\left[(\sin \theta)^{2}\left(D_{\theta} T\right)\right] \\
& +\left(D_{\rho} R\right)\left\{T+D_{\theta}\left[(\sin \theta)^{2} T\right]+(\sin \theta)^{3}\left(D_{\theta} T\right)\right\} \\
& +D_{\rho}\left(D_{\rho} R\right)(\sin \theta)^{2} T
\end{aligned}
$$

With the generating function this leads to

$$
f(\rho, \theta)=\rho^{\gamma} \mathrm{e}^{-\rho}\left[f_{1}(\sin \theta)^{\alpha-1}+f_{2}(\sin \theta)^{\alpha+1}+f_{3}(\sin \theta)^{\alpha+3}\right]
$$


where

$$
\begin{gathered}
f_{1}=-\alpha^{2}\left(\alpha^{2}-1\right) \\
f_{2}=\left(3+4 \alpha+2 \alpha^{2}\right)\left[\alpha(\alpha+1)-\gamma(\gamma+1)+2(\gamma+1) \rho-\rho^{2}\right] \\
f_{3}=-\alpha(\alpha+1)(\alpha+2)(\alpha+3)+\left(6+6 \alpha+2 \alpha^{2}\right)\left[\gamma(\gamma+1)-2(\gamma+1) \rho+\rho^{2}\right] \\
-\gamma^{2}(\gamma+1)^{2}+\left(4+12 \gamma+8 \gamma^{2}+2 \gamma^{3}\right) \rho \\
-\left(14+18 \gamma+6 \gamma^{2}\right) \rho^{2}+(8+4 \gamma) \rho^{3}-\rho^{4}
\end{gathered}
$$

Turning first to the polar component $F_{\theta}$ of the force (9), expressions (35)-(38) show that the contribution from $f(\rho, \theta)$ is symmetric in respect to the equatorial plane, whereas the contribution from the polar component $e_{\theta}^{*}$ of the electromagnetic field is antisymmetric in respect to the same plane. Therefore the integrated polar force will vanish.

The radial force component $F_{r}$ and the component $e_{r}^{*}$ of the electromagnetic field are on the other hand symmetric in respect to the equatorial plane. Therefore $F_{r}$ does not generally vanish, and from Equations (9) (26) (31) and (36) it has the form

$$
F_{r}=4 \pi \varepsilon_{0} G_{0}^{2} \int_{0}^{\infty} h(\rho) \mathrm{d} \rho
$$

where its radial force distribution becomes

$$
h(\rho)=\rho^{2 \gamma-1} \mathrm{e}^{-2 \rho}\left(F_{1} I_{2 \alpha-1}+F_{2} I_{2 \alpha+1}+F_{3} I_{2 \alpha+3}+F_{4} I_{2 \alpha+5}\right)
$$

with

$$
F_{1}=g_{1} f_{1} \quad F_{2}=g_{1} f_{2}+g_{2} f_{1} \quad F_{3}=g_{1} f_{3}+g_{2} f_{2} \quad F_{4}=g_{2} f_{3}
$$

according to Equations (32)-(33) (39)-(41).

The results of Equation (23), (31) and (40) for the parameter values $\gamma=1$ and $\alpha=1$ are illustrated in Figure 1 .

\subsection{Problems of a Self-Consistent Force Balance}

The field equations of the present theory as well as that by Maxwell give rise to linear general solutions. They can formally be derived from a nonlinear Lagrangian density, and their solutions can be combined to form a force density such as that of Equation (9).

But this is not the whole problem of a self-consistent approach. In the theory of a magnetized plasma, the electromagnetic field equations form an essential part of the force balance, but the latter also has to include the force of a pressure gradient. In the present approach, as well as in the conventional theory on elementary particles, there is a problem how to treat the particle interior. There are at least two questions to be raised in this connection:

- Can a self-consistent force balance be established when the simple Ansatz of Equation (10) is replaced by other more general forms?

- Or has an extra force field to be added to the electromagnetic one, to establish a local balance? Could a gradient of the ZPE photon pressure serve this 


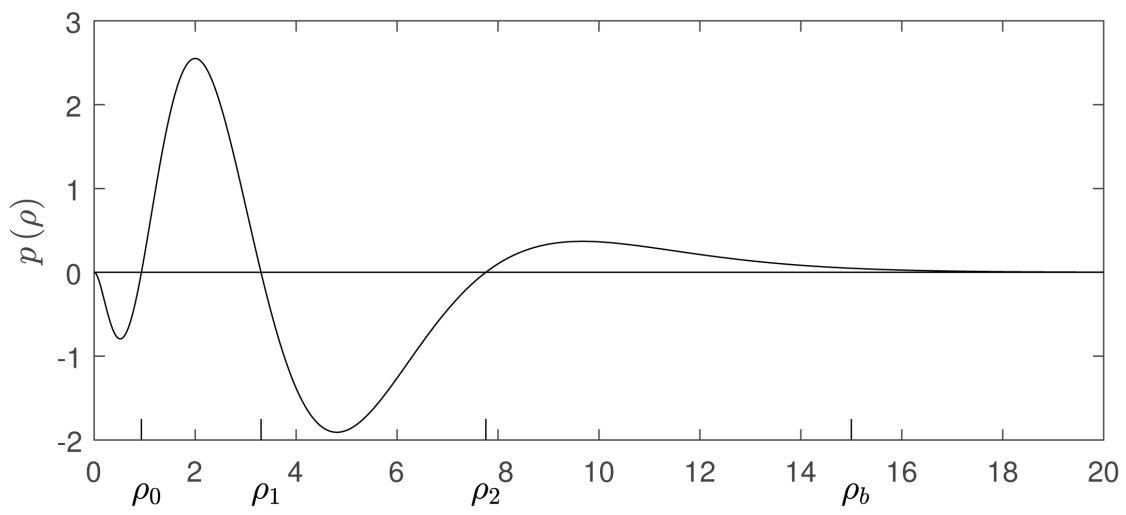

(a)

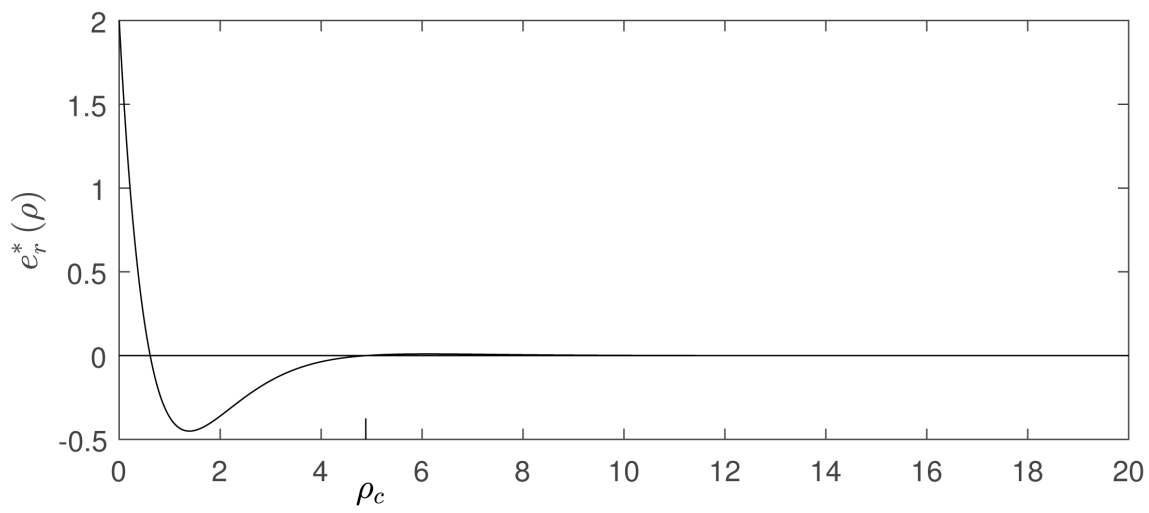

(b)

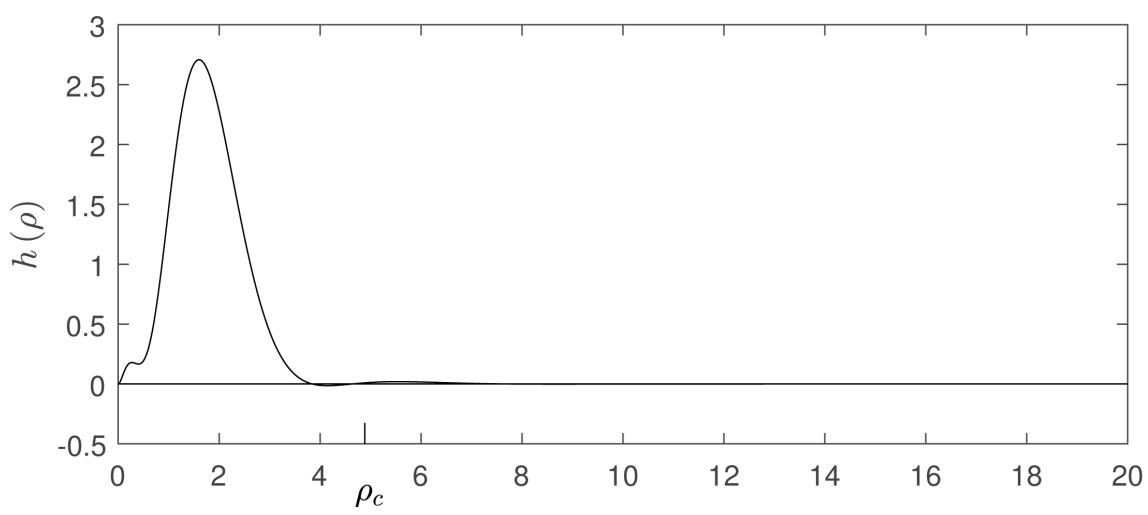

(c)

Figure 1. Normalized radial distribution for the parameter values $\gamma=1$ and $\alpha=1$. The scale of the horizontal axis is given in units of the characteristic radius $r_{0}=2.81 \times 10^{-16}$ m: (a) Charge density $p(\rho)$ having zero points $\left(\rho_{0}, \rho_{1}, \rho_{2}\right)$ and an approximate boundary at the radius $\rho_{b}$; (b) Radial electromagnetic field strength $e_{r}^{*}(\rho)$ being limited to a core having the approximate radius $\rho_{c}$; (c) Radial self-force $h(\rho)$ being limited to the core of the electromagnetic field strength.

purpose, as being represented by an energy density $f g$ defined by Equations (8) and (12), or in some other way?

These questions require more investigations, being outside the frame of the present considerations. Keeping these points in mind, the following sections will 
give a detailed example on a particle model and a related one of a neutron and proton configuration.

\section{A Tentative Quark Model of the Proton and Neutron}

The particle configuration being studied here is based on a class of solutions with similar but varying profile shapes. Here a detailed illustration will be presented, as obtained from a special choice of the parameters $\gamma$ and $\alpha$. It concerns both the features of the particle model of Section 4 and its combination into a triangular geometry representing a first attempt to make a quark model of the proton and the neutron.

\subsection{Short-Range Interaction}

Earlier results [3] [4] [6] as well as those presented in this investigation, predict the intrinsic electric charges to be much larger than the elementary charge. This will modify the interaction between two particle models of the present type. A number of points then have to be taken into account:

- The interaction has a short range, because it takes place only when the spatially limited force fields overlap.

- The interaction is not only due to the electrostatic field generated by the intrinsic charges, but there is also a partly outbalancing effect by the magnetostatic part of the electromagnetic field as shown in Section 4.2.

- The spatially distributed forces due to the intrinsic charges can interact and perturb each other during a particle collision process. This complicates a rigorous treatment. In other words, there are two different types of impact processes, namely (a) those originating from divergent generating functions which lead to point-like long-range interaction of single net sources, and (b) those originating from convergent generating functions which lead to spatially distributed shortrange interaction of multiple intrinsic sources.

- In this first investigation only the mutual effects of the unperturbed forces will be considered.

\subsection{Starting Points of the Quark Model}

Due to conventional theory, the proton and neutron consists of three quarks which are coupled in a triangular configuration [8] [9]. Here a first attempt will be made to outline a quark model, with the particle model of Section 4 forming the contribution from each quark. The net electric quark charges in the conventional model can thereby be taken into account by a compound generating function, as described in Section 2. Since the intrinsic charges are much larger than the net charges, they outbalance by far the effects of the latter in the particle interaction process.

In a first approximation, we put all the three quark masses $m$ equal to a third of the proton or neutron masses, i.e. $m=5.57 \times 10^{-28} \mathrm{~kg}$. Equation (13) then yields a characteristic radius 


$$
r_{0}=\left(h J_{m} / 2 \pi c m J_{s}\right)=6.31 \times 10^{-16}\left(J_{m} / J_{s}\right)
$$

and Equation (15) results in

$$
\left(r_{0} G_{0}\right)^{2}=h c / 2 \pi^{2} \varepsilon_{0} J_{s}=1.14 \times 10^{-15} / J_{s}
$$

As compared to the elementary charge, the relative magnitude of the intrinsic charges of either sign becomes

$$
\pm Q / e=4 \pi \varepsilon_{0}\left(G_{0} r_{0} / e\right) Q^{\prime}
$$

where $Q^{\prime}$ is the normalized intrinsic charge value to be determined in later given concrete examples.

\subsection{Illustration by a Specified Example}

Among the various but similar profile shapes represented by the generating function (10), we will choose the example given by the parameter values $\gamma=1$ and $\alpha=1$ as a first and detailed illustration. With this choice $J_{m}=17.7$ and $J_{s}=39.8$ according to Equations (14) and (16). For a mass $m$ being a third of the proton mass, Equations (42) and (43) then yield $r_{0}=2.81 \times 10^{-16} \mathrm{~m}$, $r_{0} G_{0}=5.35 \times 10^{-9} \mathrm{~m} \cdot \mathrm{V}$ and $G_{0}=1.91 \times 10^{-7} \mathrm{~V}$.

\subsubsection{Intrinsic Features of the Particle Model}

The radial distribution of space charge density given by Figure 1(a) has zero points at the normalized radii $\rho_{0}=0.93, \rho_{1}=3.3$ and $\rho_{2}=7.75$. The corresponding integrated normalized intrinsic charges are $Q_{00}^{\prime}=-0.44$, $Q_{01}^{\prime}=+3.73, Q_{12}^{\prime}=-4.92$ and $Q_{2 b}^{\prime}=+1.63$. The total intrinsic charge of each polarity is thus of the relative magnitude $\pm Q / e=19.9$ according to Equation (44). From Figure 1(a) is further seen that the normalized radial extension of the space charge configuration is $\rho_{b} \cong 15$.

The radial component $e_{r}^{*}$ of the normalized electromagnetic field strength is symmetric in respect to the equatorial plane, and the polar component $e_{\theta}^{*}$ of the same field is antisymmetric. The radial distribution of $e_{r}^{*}$ is demonstrated in Figure 1(b). Since $\boldsymbol{C} \times \boldsymbol{B}$ outbalances part of $\boldsymbol{E}$, the total field strength $\boldsymbol{E}^{*}$ only becomes noticeable in the innermost radial region of the particle model, within a core having the radius $\rho_{c}=4.5$. This core only occupies about $\left(\rho_{c} / \rho_{b}\right)^{3}=0.027$ of the particle volume, whereas about 97 percent of the same volume are in a force-free state.

The distribution of the radial electromagnetic self-force of Equation (40) is further shown in Figure 1(c). This force has been found to be positive (expansive) for a large number of choices of $\gamma$ and $\alpha$. As is also indicated in Figure 1 (b), both the self-force and the electromagnetic field strength are localized within the small core radius $\rho_{c}$.

\subsubsection{Interaction between Two Quark Particles}

In the case of a long-range interaction between two particles of charge $Q$, the obtained result $(Q / e)^{2}=396$ would imply that the related electrostatic force becomes more that two orders of magnitude larger than that between two ele- 
mentary charges. But as will be seen here, the real situation becomes different, both due to the short-range interaction between the intrinsic charges and to the partly outbalance of the electrostatic field by the magnetostatic part.

In the analysis of the short-range interaction between two particles of the present model, the volumes of Figure 1(a) are now divided into three regions. Region (1) is defined by $0 \leq \rho \leq \rho_{1}$ which includes the intrinsic charge $Q_{1}^{\prime}=Q_{00}^{\prime}+Q_{01}^{\prime}=+3.29$, Region (2) by $\rho_{1} \leq \rho \leq \rho_{2}$ with the charge $Q_{2}^{\prime}=Q_{12}^{\prime}=-4.92$, and Region (3) by $\rho_{2} \leq \rho \leq \rho_{b}$ with the charge $Q_{3}^{\prime}=Q_{2 b}^{\prime}=+1.63$. In all these cases the mutual interaction force $F_{i n}$ in Region (n), directed along the connection line between the particles, can be obtained from the charge $Q_{n}^{\prime}$ of Particle (I) interacting with the effective reversed component $\left\langle e_{r}^{*}\right\rangle_{n}$ of Particle (II). Thus

$$
F_{\text {in }}=4 \pi \varepsilon_{0} G_{0}^{2} Q_{n}^{\prime}\left(V_{s n} / V_{n}\right)\left\langle e_{r}^{*}\right\rangle_{n}\langle\cos \psi\rangle_{n}
$$

Here $V_{s n}$ is the volume occupied by the charge $Q_{n}^{\prime}$ of Particle (I), $V_{s n}$ is the part of the same volume which is overlapped by the core of Particle (II), and $\langle\cos \psi\rangle_{n}$ is an effective value related to the particle connection line.

An illustration of special interest is given by Figure 2 which concerns Region (2). In this region

$$
V_{s 2} / V_{2}=3\left(1-f_{12}\right)(\sin \beta)_{2}^{2} / 8\left(1-f_{12}^{3}\right)
$$

where $f_{12}=\rho_{1} / \rho_{2}=0.426, \quad(\sin \beta)_{n}^{2}=0.296, V_{s 2} / V_{2}=0.069,\langle\cos \psi\rangle_{2}=0.76$, and $\left\langle e_{r}^{*}\right\rangle_{2}=0.19$. This results in a maximum attraction force $\hat{F}_{i 2}=-1.98 \times 10^{3} \mathrm{~N}$. This force can be compared to the repulsive electrostatic force

$$
F_{e}=e^{2} / 4 \pi \varepsilon_{0} r_{0}^{2} \delta_{2}^{2}
$$

between two elementary charges separated by the distance $d_{2}=r_{0} \delta_{2}$ of the particle centra in Figure 2. The corresponding relative magnitude becomes

$$
\hat{F}_{i 2} / F_{e}=-\left(4 \pi \varepsilon_{0} G_{0} r_{0} / e\right)^{2} Q_{2}^{\prime}\left(V_{s 2} / V_{2}\right)\left\langle e_{r}^{*}\right\rangle_{2}\langle\cos \psi\rangle_{2} \delta_{2}^{2}
$$

With $\delta_{2}=8$ this yields $\hat{F}_{i 2} / F_{e}=-43.2$. Thus the maximum attractive force due to the intrinsic charge becomes nearly two orders of magnitude larger than the corresponding conventional and repulsive electrostatic force.

When applying Equations (45) and (46) to Region (3), the geometry corresponding to Figure 2 results in $f_{2 b}=\rho_{2} / \rho_{b}=0.517, \quad(\sin \beta)_{3}^{2}=0.19$, $V_{s 3} / V_{3}=0.040,\langle\cos \psi\rangle_{3}=0.71$, and $\left\langle e_{r}^{*}\right\rangle_{3}=0.11$. This leads to a repulsive force having the maximum value $\hat{F}_{i 3}=0.36 \times 10^{3} \mathrm{~N}$.

Finally, in the case of Region (1), the corresponding deductions become somewhat uncertain, especially in its innermost parts which correspond to small distances between the particle centra. In any case, estimations show that the related maximum force $\hat{F}_{i 3}$ becomes repulsive and of the same order as the maximum attractive force in Region (2).

It should be observed that the self-force (9) and the particle interaction force 


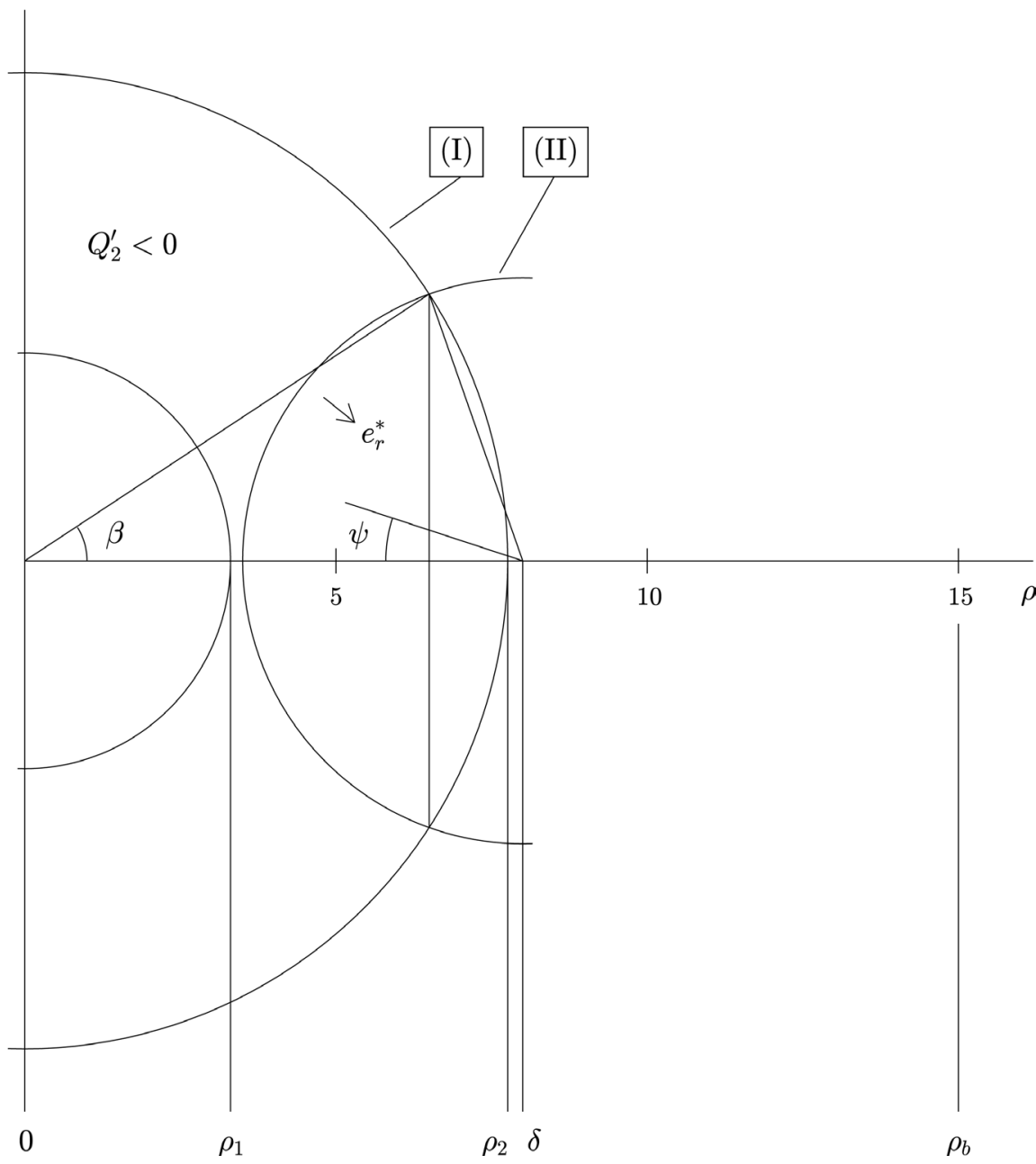

Figure 2. The situation of maximum attractive force $\hat{F}_{i 2}$ which arises at the normalized distance $\delta=8$ between the centra of particles (I) and (II). The intrinsic charge $Q_{2}^{\prime}=Q_{12}^{\prime}=-4.92$ of particle (I) in the region $\rho_{1} \leq \rho \leq \rho_{2}$ interacts with the reversed field $e_{r}^{*}$ of particle (II). The scale of the horizontal axis is given in units of the characteristic radius $r_{0}=2.81 \times 10^{-16} \mathrm{~m}$. The normalized radii $\left(\rho_{1}, \rho_{2}, \rho_{b}\right)$ are taken from Figure 1(a).

(45) are different. The former is due to the charge density (23) and the field component (29), whereas the latter arises from the density (23) of Particle (I) in combination with the reversed and displaced field component (29) of Particle (II). Even if a fully force-free state of the self-force can be attained, the particle interaction force would therefore remain to be non-zero.

The radial expansive force within the small inner core of the present model can be interpreted to exert a push on the large outer force-free shell. In its turn, this could produce an induced unbalance of the electrostatic and magnetostatic forces within the shell, to result in an integrated totally force-free state.

The distribution of the interactive force $F_{i n}$ along the radial coordinate $\rho$ in Figure 1(a) can be related to a potential $P$ where

$$
P_{n}=\int F_{i n} \mathrm{~d} r=r_{0} \int F_{i n} \mathrm{~d} \rho
$$


Then there is a potential well related to Region (2), and inner and outer potential barriers to Regions (1) and (3). An approximated picture of the radial potential distribution can then be given as follows. The boundaries between these regions are defined by the zero points $\rho_{1}$ and $\rho_{2}$ in Figure 1(a), and the related maximum forces $\hat{F}_{i 1}, \hat{F}_{i 2}$ and $\hat{F}_{i 3}$ are localized at the centra of respective regions. Consequently, the total height of the outer barrier becomes

$$
P_{3}=\frac{1}{2} \hat{F}_{i 3}\left(\rho_{b}-\rho_{2}\right) r_{0}
$$

as given by the average value $\hat{F}_{i 3} / 2$ of the force which acts along the total distance $\left(\rho_{b}-\rho_{2}\right) r_{0}$ of Region (3). The height thus becomes $P_{3}=0.36 \times 10^{-12} \mathrm{~J}=2.3 \mathrm{MeV}$. Likewise the total depth of the well becomes

$$
P_{2}=\frac{1}{2} \hat{F}_{i 2}\left(\rho_{2}-\rho_{1}\right) r_{0}
$$

Here $P_{2}=-1.24 \times 10^{-12} \quad \mathrm{~J}=-7.7 \mathrm{MeV}$.

Finally, the total height $P_{3}$ of the inner barrier becomes somewhat uncertain, but is of the same magnitude as the depth of the well.

The potential distribution $P(\rho)$ of mutual interaction at shrinking distances between the particle centra can thus be described as follows:

- At distances being somewhat larger than $\rho_{b}$ the potential becomes negligible due to this short-range interaction.

- When $\rho$ decreases to $\rho_{2}$ the first barrier of height $P_{3}=2.3 \mathrm{MeV}$ is being reached.

- A further decrease of $\rho$ to $\rho_{1}$ makes the potential decrease from $P_{3}$ by the depth $P_{2}=-7.7 \mathrm{MeV}$ of the well, down to $P_{3}+P_{2}=-5.4 \mathrm{MeV}$.

- When reaching the region below $\rho_{1}$ the potential increases again due to the inner barrier, from $P_{3}+P_{2}$ up to a level being comparable to the depth of the well.

- Due to this distribution there is a stable position of the particle interaction, near the bottom of the well.

- An impact energy of the magnitude of the well depth would be needed to release the binding between these quark particles. This is of the same magnitude as the binding energy of $8 \mathrm{MeV}$ given for the neutron according to Bethe [10].

\section{Conclusions}

The main purpose of this investigation is to elaborate a model of quarks which form triangular neutron and proton configurations. The model has the following characteristic features:

- There are equal intrinsic electric charges of both polarities.

- These intrinsic charges are an order of magnitude larger than the elementary charge e.

- The electromagnetic field has both electric and magnetic components of which the latter outbalance part but not all of the former.

- The particle model which represents each of the quarks consists of an outer 
force-free shell defined by the spatial charge distribution, and an inner core within which there are self-forces due to a nonzero electromagnetic field in combination with an intrinsic charge density. The inner core occupies only a few percent of the total particle volume.

- The interaction which arises between two such particles is of short-range character. When gradually shrinking the distance between the particle centra, a corresponding potential distribution is traversed, in the form of one outer barrier, an intermediate well, and an inner barrier.

- The well is of particular interest, because it is associated with a large attractive force. The latter is nearly two orders of magnitude larger than the corresponding repulsive force between two elementary charges.

- The deduced depth of the well is about $7.7 \mathrm{MeV}$, being nearly of the same magnitude as the binding energy $8 \mathrm{MeV}$ of the neutron.

- The obtained distribution of the barriers and the well makes a stable position of the particle distance possible, near the bottom of the well.

- The present triangular quark model of the neutron and the proton has an outer radius of about $3 \times 10^{-15} \mathrm{~m}$ and a core radius of about $10^{-15} \mathrm{~m}$. These values are comparable to the nuclear radius of $1.5 \times 10^{-15} \mathrm{~m}$ given for the neutron and the proton [10].

The results of the present investigation could thus form a first step in a unification of the electromagnetic and the strong nuclear forces.

\section{Acknowledgements}

The author is indebted to MSc Yushan Zhou for a valuable work with the computations of the present analysis. The author also thanks MSc Kerstin Holmström for an excellent transcription of the manuscript of the present paper.

\section{References}

[1] Casimir, H.B.G. (1948) Proc. K. Ned. Akad. Wet., 51, 793-795.

[2] Lamoreaux, S.K. (1997) Physical Review Letters, 78, 5-8. https://doi.org/10.1103/PhysRevLett.78.5

[3] Lehnert, B. (2013) Revised Quantum Electrodynamics. In: Dvoeglazov, V.V., Ed., Contemporary Fundamental Physics, Nova Science Publishers, Inc., New York.

[4] Lehnert, B. (2016) Journal of Modern Physics, 7, 1316-1319. https://doi.org/10.4236/jmp.2016.711117

[5] Walker, J.S. (2010) Physics. 4th Edition, Pearson International Edition, AddisonWesley, Boston, p. 1144.

[6] Lehnert, B. (2013) Progress in Physics, 3, 17-20.

[7] Lehnert, B. (2014) Progress in Physics, 10, 5-7.

[8] Ryder, L. (1996) Quantum Field Theory. 2nd Edition, Cambridge University Press, Cambridge. https://doi.org/10.1017/CBO9780511813900

[9] Riordan, M. and Zajk, W.A. (2006) The First Few Microseconds. Scientific American, 294, 34-41. https://doi.org/10.1038/scientificamerican0506-34a

[10] Bethe, H.A. (1947) Elementary Nuclear Theory. John Wiley \& Sons, Inc., New York and Chapman and Hall, Ltd., London, Ch. II and p. 118. 
Submit or recommend next manuscript to SCIRP and we will provide best service for you:

Accepting pre-submission inquiries through Email, Facebook, LinkedIn, Twitter, etc. A wide selection of journals (inclusive of 9 subjects, more than 200 journals)

Providing 24-hour high-quality service

User-friendly online submission system

Fair and swift peer-review system

Efficient typesetting and proofreading procedure

Display of the result of downloads and visits, as well as the number of cited articles Maximum dissemination of your research work

Submit your manuscript at: http://papersubmission.scirp.org/

Or contact jmp@scirp.org 